\title{
MANAGEMENT OF DUPUYTREN'S DISEASE- OUR EXPERIENCE IN A TERTIARY CARE INSTITUTE
}

\author{
Mahesh S. G1, Adarsh M. S2, Balaji R3, Mali Chetan 4 \\ ${ }^{1}$ Associate Professor, Department of Plastic Surgery, KIMS and RC, Bangalore, Karnataka, India. \\ $2^{\text {nd }}$ Year Postgraduate Student, Masters in General Surgery, KIMS and RC, Bangalore, Karnataka, India. \\ 3 Professor, Department of Plastic Surgery, KIMS and RC, Bangalore, Karnataka, India. \\ ${ }_{4}^{4}$ Assistant Professor, Department of Plastic Surgery, KIMS and RC, Bangalore, Karnataka, India.
}

ABSTRACT

\section{BACKGROUND}

Dupuytren's disease is a benign fibroproliferative disorder that causes the fingers to be drawn into the palm by formation of new tissue under the glabrous skin of the hand. This disorder causes functional limitations, but it can be treated through a variety of surgical techniques with good outcome.

Aims and Objectives- To assess the benefits of surgical procedures (Limited superficial fasciectomy and Radical superficial fasciectomy) for treatment of Dupuytren's disease of the hand including fingers (ring, middle, little, index and thumb).

\section{MATERIALS AND METHODS}

This is a prospective study. We included patients with Dupuytren's disease, who were categorised into groups receiving surgical intervention for the disease (nodules, cords and contracture bands) of the index, middle, ring, little finger, thumb and palm. Luck's criteria were considered for categorising the patients. Their records and photographs were reviewed along with histology reports. We analysed outcomes into the following categories: (1) Hand function, (2) Early objective outcomes (e.g. correction of angular deformity) and (3) Late objective outcomes (e.g. recurrence, disease progression).

Settings and Study Design- A retrospective study/ descriptive study of 30 patients who underwent surgical intervention for Dupuytren's disease for a period of five years from 2011 to 2016 at Kempegowda Institute of Medical Sciences and Research Centre, Bangalore were considered in this study.

\section{RESULTS}

In our study of 30 patients, 29 patients were males and majority were in the age group of 45 to 70 years. We did not encounter any patients with Plantar Fasciitis/ Peyronie's disease or any related disease complex, but 6 patients had Diabetes Mellitus. There was positive family history in 3 patients with bilateral disease noted in 18 patients. Hand function improved in majority (83.33\%) of patients and we were able to correct angular deformities. Disease recurrence was seen in 3 patients with progression seen in 3 patients. We did not have any neurovascular complications in our study.

\section{CONCLUSION}

Understanding current treatment patterns, we were able to achieve good outcome in all our patients with single digit affliction and unilateral disease. Patients presenting with bilateral advanced disease were left with residual contracture. Overall improvement was observed in all our patients with the current treatment modalities (Limited superficial fasciectomy and Radical superficial fasciectomy) coupled with concurrent continuous and aggressive physiotherapy.

\section{KEY WORDS}

Dupuytren's Disease, Palmar Nodule, Finger Contracture.

HOW TO CITE THIS ARTICLE: Mahesh SG, Adarsh MS, Balaji R, et al. Management of Dupuytren's disease- our experience in a tertiary care institute. J. Evolution Med. Dent. Sci. 2018;7(24):2904-2908, DOI: 10.14260/jemds/2018/654

\section{BACKGROUND}

Dupuytren's disease[1] is a flexion contracture of the hand due to palmar fibromatosis,[2] in which the fingers bend towards the palm and cannot be fully extended (straightened). It is an inherited proliferative connective tissue disorder that involves the hand's palmar fascia.[3] It is named after Baron Guillaume Dupuytren, the surgeon who described an operation to correct the affliction. Dupuytren's contracture has been suggested as being Viking in origin

'Financial or Other Competing Interest': None.

Submission 06-04-2017, Peer Review 24-01-2018,

Acceptance 31-01-2018, Published 11-06-2018.

Corresponding Author:

Dr. Mahesh $S$. $G$

Flat No. 701, M-Block,

Brigade Gateway, Malleshwaram,

Bangalore-55.

E-mail: drmaheshg2001@gmail.com

DOI: $10.14260 /$ jemds $/ 2018 / 654$

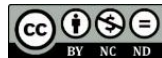

(Hueston, 1985).[4,5] It is common amongst Caucasians and wherever they have settled their propensity to this condition is evident. It has also been reported among Negroes, [2,6,7] both African and migrant (Plasse, 1979; Furnas, 1979; Zaworsky and Mann, 1979),[6,8] Vietnamese (Maes, 1979) ${ }^{[9]}$ and Chinese (Chow et al, 1984).[10] Studies have shown an appreciable incidence of Dupuytren's disease among the Japanese (Egawa et al, 1985).[4] There are reports of Dupuytren's disease among the patients of Indian origin. James (1985) reviewing the genetic pattern of Dupuytren's disease states[11,12]- "It would seem probable that Dupuytren's does not occur among the Negroes, Chinese or Asiatic Indians of pure stock, or if later cases become known they will be exceptional since they are unknown at present to surgeons working amongst these races." We have come across 30 patients from India operated upon in our department for Dupuytren's disease and they are the subject of this paper. The traditional role of surgery in the treatment of Dupuytren's disease is being challenged by less invasive treatment options, specifically percutaneous needle 
fasciotomy and more recently collagenase injection, partial fasciectomy and dermofasciectomy.[13,14,15] Nonetheless, surgery maintains an important place in the management of this condition. For many surgeons and in many situations, for example multiple digit involvement, recurrent disease,[16] severe contractures and where collagenase is unavailable or unaffordable surgery remains the primary treatment.

\section{MATERIALS AND METHODS}

This is a prospective study with 30 patients from India with Dupuytren's disease of the age group 45 - 70 yrs. were included in this study. There were 29 male patients and 1 female patient, of which majority were manual labourers. 6 patients were diabetic with positive family history noted in 3 patients. None gave history of epilepsy/ alcoholism. 18 patients had bilateral disease at the time of presentation or the other hand became involved subsequently and 12 patients presented with unilateral disease. 17 patients with $>30$ degrees of MCP joint contracture and any degree of PIP joint contracture, more than two digits involvement and with a positive table top test underwent radical superficial fasciectomy. 13 patients with only 1 or 2 digits involvement and with nodules (painless, commonly located within ulnar aspect of palm along ring finger at the level of distal palmar crease and at PIP joint level) underwent limited superficial fasciectomy.[8,17,18] All patients who were fit were operated under General anaesthesia with an upper limb tourniquet to limit the blood supply to the involved limb. Patients who were unfit were operated under regional anaesthesia (limb blocks). Zigzag plasty incision[19,17,20] along the palmar creases depending on the number of digits involved with an oblique incision made over the digits was considered. Adequate flap was raised to maintain vascularity. However, in radical superficial fasciectomy both the diseased and even normal-appearing fascia is excised, but in limited superficial fasciectomy only the diseased cord and fascia is excised. Excision was very precise to spare the neurovascular bundles in both the procedures.[21,17,20] The operative findings in all the cases were those of Dupuytren's disease with bands causing flexion contracture or restriction of extension.

\section{Objective of the Study}

Dupuytren's disease, though considered an inherited connective tissue disorder is a multifactorial disease.

\section{The Main Objectives of the Study}

- Correction of deformity.

- Reducing disability.

- Restoring hand function.

Enabling patient to carry out daily activities without difficulty through surgical management, i.e. Limited Superficial Fasciectomy and Radical Superficial Fasciectomy. Surgery remains the mainstay of treatment in our study. Study is carried out to establish the detailed pattern of Dupuytren's disease among Indians, although considered a rare disorder among Indians.

\section{Post-Operative Care}

Post surgery a light dressing is applied and the surgical dressing is removed after five days. Active and passive flexion and extension exercises are commenced in the immediate post-operative period with the emphasis on maintaining full extension, especially at the PIP joint. A night extension splint[22,23] is usually worn for six weeks to aid the maintenance of extension. On the fourteenth day, the sutures were removed, and any open areas of the wound were covered with small non-adhesive dressings. Exercises were continued until full range of motion and strength was regained. However, an early recurrence at the PIP Joint contracture can be reversed by using a dynamic extension splint.

MCPJ- Metacarpophalangeal Joint, PIPJ- Proximal Interphalangeal Joint

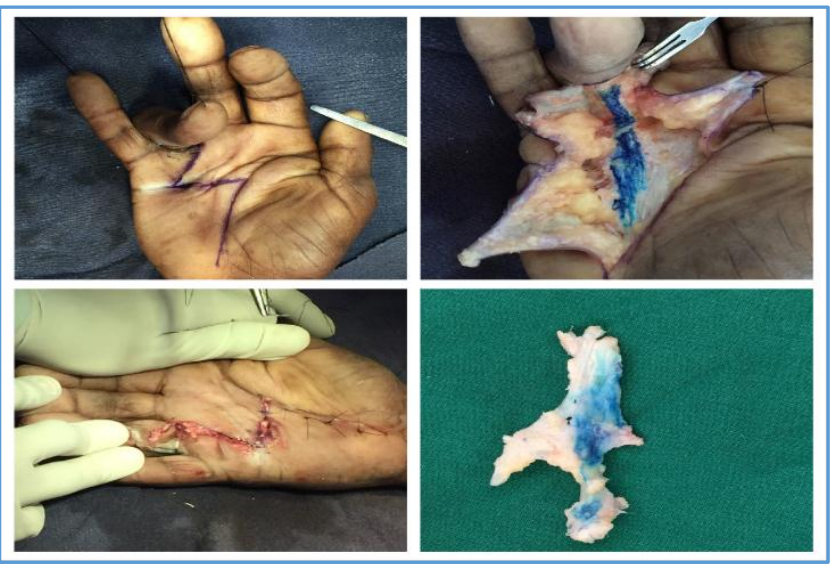

Figure 1. Limited Superficial Fasciectomy

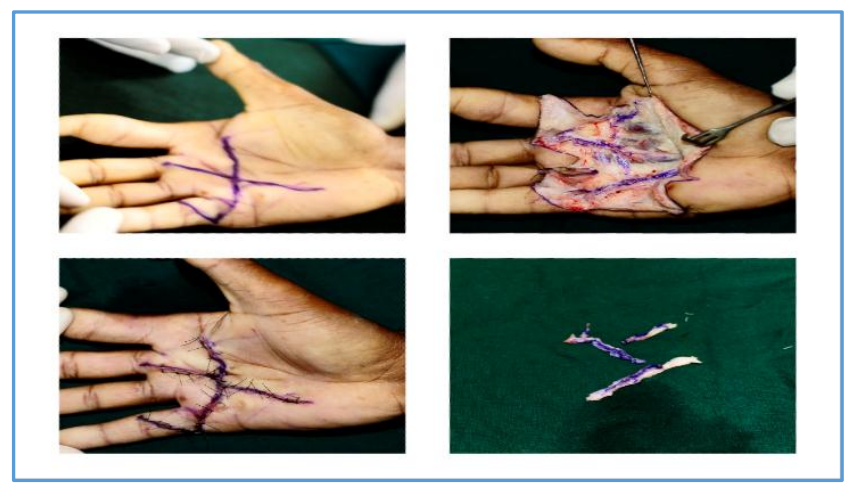

Figure 2. Radical Superficial Fasciectomy

\section{Data Entry and Analysis}

The data was entered in Microsoft Excel and analysed using EpiData analysis software V2.2.2.186. The continuous variables such as age, range of joint contracture and movements before and after surgery and improvements in range of movements were summarised as mean (SD). The categorical variables including gender, side of lesion, occupation, clinical presentation and type of surgery were summarised as proportions. The improvement in range of movements before and after surgery was compared using paired ' $\mathrm{t}$ ' test. $\mathrm{P}$ value of $<0.05$ was considered as statistically significant.

\section{RESULTS}

\begin{tabular}{|c|c|c|c|}
\hline & Mean Age & $\begin{array}{c}\text { Standard } \\
\text { Deviation }\end{array}$ & $\begin{array}{c}\text { Total Number of } \\
\text { Participants }\end{array}$ \\
\hline Age in years & 56.9 & 6.6 & 30 \\
\hline \multicolumn{3}{|c|}{ Table 1 } \\
\hline
\end{tabular}


In our study of 30 patients presenting with Dupuytren's disease, mean age group with SD (Standard Deviation) is $56.9 \pm 6.6$ years. However, majority $(43.3 \%)$ of patients presented with Dupuytren's disease during the $6^{\text {th }}$ decade.

\begin{tabular}{|c|c|c|}
\hline Gender & Number & Percentage \\
\hline Male & 29 & 96.7 \\
\hline Female & 1 & 3.3 \\
\hline Total & $\mathbf{3 0}$ & $\mathbf{1 0 0}$ \\
\hline \multicolumn{3}{|c|}{ Table 2 } \\
\hline
\end{tabular}

Among 30 patients in our study 29 patients were males, as majority were manual labourers and there was only 1 female patient who was a part of our study group.

\begin{tabular}{|c|c|c|}
\hline Hand Involved & Number & Percentage \\
\hline Unilateral & & \\
Dominant hand & 8 & 26.6 \\
Non-dominant hand & 4 & 13.3 \\
\hline Bilateral & 18 & 60.0 \\
\hline Total & $\mathbf{3 0}$ & $\mathbf{1 0 0}$ \\
\hline
\end{tabular}

\section{Table 3. Hand Involvement of the Study Participants}

Of the 30 patients in the study B/L (bilateral) involvement of the disease was noted in $18(60 \%)$ of patients and unilateral hand involvement was noted in $12(40 \%)$ of patients. Of the 12 patients, $8(26.6 \%)$ patients had involvement of the dominant hand and 4 patients had the involvement of the non-dominant hand (13.3\%). However, usually considering the dominant hand to be the right hand and non-dominant hand being the left hand in majority of patients.

\begin{tabular}{|c|c|c|}
\hline Occupation & Number & Percentage \\
\hline Accountant & 1 & 3.3 \\
\hline Carpenter & 2 & 6.7 \\
\hline Civil Servant & 1 & 3.3 \\
\hline Drilling Machine Operator & 2 & 6.7 \\
\hline Gardener/ Horticulture & 4 & 13.3 \\
\hline Lecturer & 2 & 6.7 \\
\hline Machine Operator & 3 & 9.9 \\
\hline Manual Labourer & 11 & 36.7 \\
\hline $\begin{array}{l}\text { Retired Lecturer/ Retired } \\
\text { Manual Worker }\end{array}$ & 4 & 13.4 \\
\hline Total & 30 & 100 \\
\hline
\end{tabular}

In our study we encountered $36.7 \%$ patients who were manual labourers, $13.3 \%$ patients were gardeners mostly requiring a stiff gripping action and $13.4 \%$ patients were retired lecturers requiring a fine pincer grip for carrying out their daily activities. Common impairment in patients in our study was in hand shaking, fitting of gloves, writing/ typing and in grasping large objects.

\begin{tabular}{|c|c|c|c|c|}
\hline \multirow{2}{*}{ Side } & \multicolumn{2}{|c|}{ Dominant Hand } & \multicolumn{2}{c|}{$\begin{array}{c}\text { Non-Dominant } \\
\text { Hand }\end{array}$} \\
\cline { 2 - 5 } & No. & $\mathbf{\%}$ & No. & $\mathbf{\%}$ \\
\hline Contraction band & 23 & 88.5 & 16 & 69.6 \\
\hline Nodules & 3 & 11.5 & 7 & 30.4 \\
\hline Total & $\mathbf{2 6}$ & $\mathbf{1 0 0}$ & $\mathbf{2 3}$ & $\mathbf{1 0 0}$ \\
\hline Table 5. Clinical Presentation of the Study Participants \\
\hline
\end{tabular}

Of the 30 patients in our study, unilateral hand involvement was noted in 11 patients and bilateral hand involvement was noted in 19 patients considering a total of 49 hands. Contracture band was noted in $23(88.5 \%)$ patients in the dominant hand and in $16(69.6 \%)$ patients in the nondominant hand involving single or multiple digits depending upon the extent of disease.

Nodules were noted in $3(11.5 \%)$ patients in dominant hand and 7 (30.4\%) patients in the non-dominant hand.

\begin{tabular}{|c|c|c|c|}
\hline \multirow{2}{*}{ Hand } & Joint & $\begin{array}{c}\text { Mean Joint } \\
\text { Contracture } \\
\text { Angle }\end{array}$ & $\begin{array}{c}\text { Standard } \\
\text { Deviation }\end{array}$ \\
\hline \multirow{2}{*}{\begin{tabular}{c} 
Dominant hand \\
\cline { 2 - 4 }
\end{tabular}} & MCPJ (N=26) & 133.3 & 20.7 \\
\cline { 2 - 4 } & PIPJ (N=23) & 100.4 & 18.5 \\
\hline $\begin{array}{c}\text { Non-dominant } \\
\text { hand }\end{array}$ & MCPJ (N=17) & 102.6 & 12.9 \\
\cline { 2 - 4 } & PIPJ (N=17) & 102.6 & 12.7 \\
\hline \multicolumn{3}{|c|}{ Table 6 } \\
\hline
\end{tabular}

\begin{tabular}{|c|c|c|c|c|}
\hline \multirow{2}{*}{ Side } & \multicolumn{2}{|c|}{$\begin{array}{c}\text { Dominant } \\
\text { Hand }\end{array}$} & \multicolumn{2}{|c|}{$\begin{array}{c}\text { Non-Dominant } \\
\text { Hand }\end{array}$} \\
\cline { 2 - 5 } & No. & $\mathbf{\%}$ & No. & $\mathbf{\%}$ \\
\hline $\begin{array}{c}\text { Radical Superficial } \\
\text { Fasciectomy }\end{array}$ & 17 & 68.0 & 13 & 56.5 \\
\hline $\begin{array}{c}\text { Limited Superficial } \\
\text { Fasciectomy }\end{array}$ & 8 & 32.0 & 10 & 43.5 \\
\hline Total & $\mathbf{2 5}$ & $\mathbf{1 0 0}$ & $\mathbf{2 3}$ & $\mathbf{1 0 0}$ \\
\hline
\end{tabular}

Table 7. Type of Surgery among the Study Participants

Of the 25 dominant hands, 17 (68\%) underwent radical superficial fasciectomy and 8 (32\%) underwent limited superficial fasciectomy. Of the 23 non-dominant hands, 13 (56.5\%) underwent radical superficial fasciectomy and 10 (43.5\%) underwent limited superficial fasciectomy.

\begin{tabular}{|c|c|c|c|c|c|c|c|c|}
\hline \multirow[t]{2}{*}{ Side } & \multirow{2}{*}{$\begin{array}{l}\text { Type of } \\
\text { Joint }\end{array}$} & \multirow[t]{2}{*}{ Procedure } & \multirow{2}{*}{$\begin{array}{c}\text { No. of } \\
\text { Participants }\end{array}$} & \multicolumn{2}{|c|}{\begin{tabular}{|c|} 
Range of Movements in \\
Joint before Surgery
\end{tabular}} & \multicolumn{2}{|c|}{\begin{tabular}{|l|} 
Range of Movements \\
in Joint after Surgery
\end{tabular}} & \multirow{2}{*}{$\begin{array}{c}\mathbf{P} \\
\text { value* }\end{array}$} \\
\hline & & & & Mean & SD & Mean & SD & \\
\hline \multirow{4}{*}{$\begin{array}{c}\text { Dominant } \\
\text { hand }\end{array}$} & \multirow{2}{*}{ MCPJ } & Radical Superficial Fasciectomy & 17 & 124.4 & 4.1 & 164.7 & 5.7 & $<0.001$ \\
\hline & & Limited Superficial Fasciectomy & 8 & 146.2 & 4.9 & 173.7 & 5.2 & $<0.001$ \\
\hline & \multirow{2}{*}{ PIPJ } & Radical Superficial Fasciectomy & 17 & 97.5 & 1.7 & 157.1 & 6.6 & $<0.001$ \\
\hline & & Limited Superficial Fasciectomy & 5 & 98.0 & 4.5 & 161.0 & 6.5 & $<0.001$ \\
\hline \multirow{4}{*}{$\begin{array}{c}\text { Non- } \\
\text { dominant } \\
\text { hand }\end{array}$} & \multirow{2}{*}{ MCPJ } & Radical Superficial Fasciectomy & 13 & 115.0 & 35.4 & 166.5 & 6.9 & $<0.001$ \\
\hline & & Limited Superficial Fasciectomy & 10 & 133.0 & 48.8 & 177.0 & 4.8 & 0.012 \\
\hline & \multirow{2}{*}{ PIPJ } & Radical Superficial Fasciectomy & 13 & 99.2 & 10.6 & 160.4 & 6.9 & $<0.001$ \\
\hline & & Limited Superficial Fasciectomy & 2 & 107.5 & 17.7 & 175.0 & 0.0 & 0.117 \\
\hline
\end{tabular}

Standard deviation $=\mathrm{SD}$, *Paired ' $\mathrm{t}$ ' test 
In the dominant hand, of the 17 hands undergoing radical superficial fasciectomy mean improvement in contracture angle at MCP joint is $40.3 \pm 1.6^{\circ}$ and at PIP joint is $59.5 \pm 4.9^{\circ}$ with a p-value $<0.001$ considering it to be statistically significant.

In the dominant hand, of the 8 hands undergoing limited superficial fasciectomy mean improvement in contracture angle at the MCP joint is $27.5^{\circ}$ and of the 5 hands with PIP joint involvement improvement in contracture angle is $63^{\circ}$.

In the non-dominant hand, of the 13 patients undergoing radical superficial fasciectomy mean improvement in contracture angle at the MCP joint is $51.5^{\circ}$ and at the PIP joint is $61.2 \pm 3.7^{\circ}$ with a P-value $<0.001$ which is statistically significant.

In the non-dominant hand of the 10 patients undergoing limited superficial fasciectomy, mean improvement in contracture angle at the MCP joint is $44^{\circ}$ and of the 2 patients with PIP joint involvement mean improvement in the contracture angle is about $67.5^{\circ}$.

\begin{tabular}{|c|c|c|c|c|c|}
\hline & 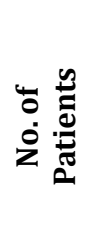 & 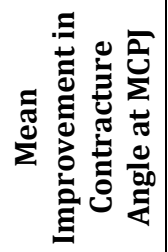 & 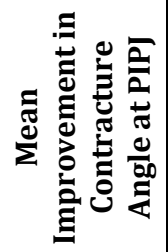 & 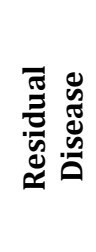 & 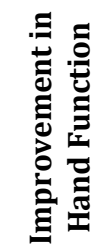 \\
\hline RSF & 17 & $45.90^{\circ}$ & $60.35^{\circ}$ & 0 & $100 \%$ \\
\hline LSF & 13 & $35.75^{\circ}$ & 65.250 & 3 & $76.9 \%$ \\
\hline \multicolumn{6}{|c|}{ Table 9} \\
\hline
\end{tabular}

Of the 17 patients undergoing radical superficial fasciectomy, mean improvement in the contracture angle (considering both the dominant and non-dominant hand) at the MCP joint is $45.90^{\circ}$ and at the PIP joint is $60.35^{\circ}$ with no residual disease and with an overall improvement in the hand function being $100 \%$ in a follow-up period of 1 year.

of the 13 patients undergoing limited superficial fasciectomy, mean improvement in the contracture angle (considering both the dominant and non-dominant hand) at the MCP joint is $35.75^{\circ}$ and at the PIP joint is $65.25^{\circ}$ with residual disease noted in 3 patients and with an overall improvement in the hand function being $76.90 \%$, in a followup period of 1 year.

No neurovascular complications were encountered in our study, though minor post-operative complications like haematoma, wound infection and flap necrosis were noted in 3 patients in either procedures. Only one patient, who underwent radical superficial fasciectomy had some scar hypertrophy on follow-up, but this did not restrict movement.

\section{DISCUSSION}

Dupuytren's disease, off late few cases are being encountered both in north India[7] (Govila, 1988) and from south India (Venkataswami, 1988). It has been suggested that Dupuytren's disease is due to an autosomally dominant gene among the Caucasian races (Ling, 1963),[4] although external factors such as alcoholism, trauma, manual labour and inactivity from paralysis have long been suspected as factors leading to contracture in a predisposed person. Epileptics are known to have a high incidence of Dupuytren's contracture (Skoog, 1948; Hueston, 1963; James, 1968).[21,24,25]
We believe that Dupuytren's disease in patients from the Indian sub-continent, though uncommon does occur and has essentially the same presentation, distribution and progression as amongst Caucasians. Further study in a larger number of patients is needed to establish the detailed pattern of Dupuytren's disease among Indians.

In our study of 30 patients, 29 patients were males and 1 female patient indicating that Indian women are also susceptible to Dupuytren's disease. It is possible that the presence of a mild contracture in a woman in her fifties or sixties does not overcome her natural reticence to seek treatment. In our study majority of the patients were in the age group of 45 to 70 years, where most of them had engaged in manual work; however, manual labour has generally been discounted as a direct cause of Dupuytren's contracture. There was no history of epilepsy amongst our patients and only $6(20 \%)$ were diabetic. A similar incidence of diabetes has been found by Venkataswami (1988), which is hardly exceptional in this age group.

\section{CONCLUSION}

We have shown from our study that good results can be achieved with both limited and radical superficial fasciectomies depending on the extent and severity of the disease. Close post-op follow-up with good supervised physiotherapy will help in maintaining good results as well as preventing recurrences in the post-operative period.

Understanding current treatment patterns, we were able to achieve good outcome in all our patients with single digit affliction and unilateral disease. Patients presenting with bilateral advanced disease were left with residual contracture.[16] Overall improvement was observed in all our patients with the current treatment modalities (limited superficial fasciectomy and radical superficial fasciectomy) coupled with concurrent continuous and aggressive physiotherapy.[12,9,26]

\section{REFERENCES}

[1] Van Rijssen AL, Gerbrandy FSJ, Ter Linden H, et al. A comparison of the direct outcomes of percutaneous needle fasciectomy and limited fasciectomy for dupuytren's disease: a 6-week follow-up study. J Hand Surg Am 2006;31(5):717-25.

[2] Zaworski RE, Mann RJ. Dupuytren's contractures in a black patient. Plastic and Reconstructive Surgery 1979;63(1):122-4.

[3] Becker GW, Davis TR. The outcome of surgical treatments for primary Dupuytren's disease - a systematic review. J Hand Surg Eur Vol 2010;35(8):623-6.

[4] Egawa T, Horiki A, Senrui H. Dupuytren's contracture in Japan. In: Hueston JT, Tubiana R, eds. Dupuytren's disease. $2^{\text {nd }}$ edn. Edinburgh: Churchill Livingstone 1985: 10@103.

[5] Hueston JT. Dupuytren's contracture. Edinburgh: Livingstone 1963: p. 108.

[6] Furnas DW. Dupuytren's contractures in a black patient in East Africa. Plastic and Reconstructive Surgery 1979;64(2):250-1.

[7] Govila A. Personal communication. 1988.

[8] Plasse JS. Dupuytren's contractures in a black patient. Plastic and Reconstructive Surgery 1979;64(2):250. 
[9] Maes J. Dupuytren's contracture in an oriental patient. Plastic and Reconstructive Surgery 1979;64(2):251.

[10] Chow SP, Luk KD, Kung TM. Dupuytren's contracture in Chinese. A report of three cases. Journal of the Royal College of Surgeons of Edinburgh 1984;29(1):49-51.

[11] James JIP. The genetic pattern of Dupuytren's disease and idiopathic epilepsy. In: Hueston JT, Tubiana R, eds. Dupuytren's Disease. 2nd edn. Edinburgh: Churchill Livingstone 1985: p. 9499.

[12] Ling RSM. The genetic factor in Dupuytren's disease. Journal of Bone and Joint Surgery 1963;45(4):709-18.

[13] Mavrogenis AF, Spyridonos SG, Ignatiadis IA, et al. Partial fasciectomy for Dupuytren's contractures. J Surg Orthop Adv 2009;18(2):106-10.

[14] Tonkin MA, Burke FD, Varian JP. Dupuytren's contracture: a comparative study of fasciectomy and dermofasciectomy in one hundred patients. J Hand Surg Br 1984;9(2):156-62.

[15] Coert JH, Nerin JP, Meek MF. Results of partial fasciectomy for Dupuytren disease in 261 consecutive patients. Ann Plast Surg 2006;57(1):13-7.

[16] Citron ND, Nunez V. Recurrence after surgery for Dupuytren's disease: a randomized trial of two skin incisions. J Hand Surg Br 2005;30(6):563-6.

[17] Robbins TH. Dupuytren's contracture: the deferred Zplasty. Annals of the Royal College of Surgeons of England 1981;63(5):357-8.

[18] Skoog T. Dupuytren's contracture. Acta Chirurgica Scandinavica 1948;96(Suppl 139): 1-190.
[19] Bruner JM. The zig-zag volar-digital incision for flexor tendon surgery. Plast Reconstr Surg 1967;40(6):5714.

[20] Wolfe SW, Hotchkiss RN, Pederson WC, et al. Green's operative hand surgery. $6^{\text {th }}$ edn. Churchill Livingstone 2011.

[21] Denkler K. Surgical complications associated with fasciectomy for Dupuytren's disease: a 20-year review of the English literature. Eplasty 2010;10:e15.

[22] Kemler MA, Houpt P, Van Der Horst CM. A pilot study assessing the effectiveness of postoperative splinting after limited fasciectomy for Dupuytren's disease. J Hand Surg Eur Vol 2012;37(8):733-7.

[23] Larson D, Jerosch-Herold C. Clinical effectiveness of post-operative splinting after surgical release of Dupuytren's contracture: a systematic review. BMC Musculoskelet Disord 2008;9:104.

[24] Early PF. Population studies in Dupuytren's contracture. Journal of Bone and Joint Surgery 1962;44-B(3):602-13.

[25] Skoff HD. The surgical treatment of Dupuytren's contracture: a synthesis of techniques. Plast Reconstr Surg 2004;113(2):540-4.

[26] Khashan M, Smitham PJ, Khan WS, et al. Dupuytren's disease: review of the current literature. Open Orthop J 2011;5(Suppl 2):283-8. 